\title{
Effect of foot massage on postoperative pain and vital signs in breast cancer patient
}

\author{
Salwa Hagag Hussien Abdelaziz, Hala Ezzat Mohammed \\ Medical surgical nursing department, faculty of nursing, Cairo University, Cairo, Egypt. \\ Correspondence: Salwa Hagag Hussien Abdelaziz. Address: 15 Mahmoud Mishreef Street, Tura Elassment, Maadi City, \\ Cairo. Email: nadakimo2005@yahoo.com or abdulazizSH@saadcollege.edu.sa
}

Received: February 9, 2014

DOI : $10.5430 /$ jnep.v4n8p115
Accepted: May 25, 2014

URL: http://dx.doi.org/10.5430/jnep.v4n8p115

\section{Abstract}

Background: Pain is a common sequela of surgery and foot massage has been advocated as an effective and easy technique that can be applied independently by nurses to patients in postoperative period to relive pain intensity. It involves a simple technique, costs little and requires no special equipment. The aim of this study is to evaluate the effect of foot massage on postoperative pain and vital sign parameters in postoperative breast cancer patients.

Methods: A quasiexperimental design was used to investigate any causality between foot massage and postoperative pain with a total of 60 breast cancer patients $(n=30$ in the control group who received only analgesic treatment and $n=30$ in the experimental group who received analgesic treatment plus foot massage). A structured questionnaire was developed by the researcher to collect data related to participants' characteristics such as age, level of education, type of surgery and usage of Non-steroidal Anti Inflammatory Drugs [NSAIDs]. Following an initial complaint from patients that they were experiencing post-operative pain the pain intensity level was assessed by a Visual Analogue Scale [VAS] as a baseline and after 60 minutes and 120 minutes following foot massage. Vital signs were assessed using the same time intervals.

Findings: When analyzing pain levels over time a significant difference was found between both groups with the mean pain level of the experimental group who had experienced foot massage as an adjunct to analgesia being noted to be lower than that of the control group. A statistically significant reduction of systolic and diastolic blood pressure in both groups was also observed but a higher reduction was observed in the experimental group $(P<.001)$ whilst the respiratory rate remained unchanged in both groups.

Conclusion: Foot massage is an effective modality in helping to relieve postoperative pain among women who have been treated with surgery for breast surgery. To promote and improve the quality of foot massage in clinical settings, regular meeting should be held by health team members to discuss the potential positive clinical outcomes, patient benefits and methods of implementation and audit of this technique. Consideration should be given by schools of nursing to include this technique within their nursing curriculum, through the integration of the complementary therapy foot massage technique as part of the considerations on the fundamentals of surgical nursing theory and practice.

\section{Key words}

Foot massage, Postoperative pain, Breast cancer, Complementary and alternative therapy

\section{I ntroduction}

One of the most critical patient complaints in modern oncological care is that of cancer related pain. Pain is also is the main reason encountered by hospitalized patients in general and surgical patients in particular and a study carried out to assess 
the prevalence of postoperative pain found that $41 \%$ of patients after surgery experienced moderate to severe pain despite the drugs and anesthetic techniques available ${ }^{[1]}$.

In many countries massage is increasingly playing an important part as an adjunct to traditional medicine as a method of pain relief. Foot massage has a long historical pedigree having been identified in both ancient Chinese and Egyptian tradition its use even being noted in hieroglyphics and the use of massage therapy [MT] has been practiced since the age of pharaohs. In fact, it is proposed that it can help cancer patients after surgery as they seek to manage their symptoms and perform tasks associated with daily living ${ }^{[2,3]}$. Breast cancer is the most common tumour among women In Egypt, representing $18.9 \%$ of the total cancer cases among Egypt National Cancer Institute [NCI] series of 10,655 patients recorded during $2001^{[4]}$.

MT incorporates a wide variety of techniques such as effleurage, petrissage, tapotement and friction and many studies have reported that the application of these techniques are effective in relieving pain level by approximately $50 \%$ for all symptoms. Indeed the techniques of MT has been demonstrated to increase circulation, stimulate venous and lymphatic drainage, improve muscle tissue metabolism and elasticity, and enhance relaxation through increased parasympathetic and reduced sympathetic nervous system activity ${ }^{[5]}$. Furthermore MT has a biological advantage whereby it increasing serotonin and dopamine whilst decreasing stress hormone levels that are found in breast cancer patients following MT.

\subsection{What is massage therapy?}

MT is defined as a manipulation of soft tissues by hands to generate positive effects on the function of various systems of the body. In addition it involves the application of combinations of specialized strokes, rubs, and the application of pressure in varying intensity to the soft tissues of the body in order to relive postoperative pain in cancer patients ${ }^{[6,7]}$.

\subsection{Benefits of Foot Massage (FM)}

Within the literature it is mentioned that FM has many benefits to cancer patients because it helps in controlling pain by closing gates in the posterior horns of the spinal cord and block the passage of noxious (i.e. pain) stimuli to CNS ${ }^{[8]}$. Also it has postulated that FM has a beneficial effect of decreasing anxiety and stress levels through elevating the body's dopamine level ${ }^{[9]}$. It has also been suggested that FM can help to improve both circulatory and the bodily functions helping them to be naturally turned into the body's homeostasis. However, whilst FM has been investigated in various studies for its therapeutic intervention effect on minimizing symptoms in cancer patients it has never the less been difficult to highlight firm conclusions in this regard ${ }^{[7]}$. Recently it has been stated that the effect of FM on relieving acute postoperative pain needs a critical analysis ${ }^{[10]}$. It is also mentioned in the literature that it is important to increase the number of studies to determine the effectiveness of FM and other non-pharmacological methods so as to better understand their place in relieving pain among postoperative cancer patients ${ }^{[1]}$.

\subsection{Mechanism of FM in relieving post operative pain}

Following surgery tissue damage naturally leads to pain which increases chemical mediators that include prostaglandin, histamine, protone, cytokines, bradykinin, serotonin and neuropeptides to stimulate local pain sensation ${ }^{[12]}$. Using FM large nerve fibers and dermatome layers are stimulated by massage through tactile and pressure receptors which transmit the nerve impulse to the CNS. The gate control system in the dorsal horn is activated through the inhibitory interneuron which is responsible to activate the gate control system in the dorsal horn, thus closing the gate which prevents transmission of pain massage to the brain ${ }^{[13]}$.

\subsection{Nurses role in managing pain using FM}

Nurses have a significant role to play in controlling and relieving postoperative pain using different modalities such as FM, reflexology, therapeutic touch and other mind-body techniques ${ }^{[14]}$ and the literature suggests that nurses can provide more effective postoperative pain management by increasing the use of $\mathrm{FM}^{[11]}$. So it is important to provide nurses and pain management personnel with scientific information about the effectiveness of FM and its uses as an essential part of an integrative approach to the management of breast cancer pain among postoperative patients. Active participation of 
surgical nurses in the judicious use of FM to relieve pain in collaboration with a multidisciplinary team is possible if they are well trained in using foot massage as a method of relieving pain during their initial formation. It is further recommended in the literature that increasing the number of studies on FM in order to evaluate the reliability and effectiveness of its technique may well positively influence both patient satisfaction and nursing practice ${ }^{[15]}$. As is always the case in using any therapeutic intervention nurses must be aware of potential contraindications of applying FM so as to avoid any harmful effects of this technique. For example it has been reported that FM is not recommended for the following patients ${ }^{[16]}$ :

Patients with coagulation disorders or low platelet count; patients using medications such as coumadin, Acetylsalicylic acid and heparin; patients who have metastases to bone and those who have open wounds ${ }^{[6]}$. Added that skin infection and vein thrombosis are contraindicated for patient receiving FM. Furthermore no evidences suggest that MT can spread cancer although avoiding direct pressure over a tumor is a sensible precaution.

\subsection{Common techniques used for FM}

The most common technique used for foot massage is petrissage in which lifting, squeezing, wringing or kneading of soft tissues is used to stimulate deep muscle and to increase circulation through the associated manipulations ${ }^{[16]}$. Additionally the technique of effleurage which is a lighter technique performed with the tips of the fingers in a circular pattern over one part of the body or in long strokes over the back or an extremity is also used on occasions. Another technique used for foot massage is the application of friction involving the application of penetrating pressure with the fingertips to reduce muscle spasm. This technique does not have to be particularly "deep" (intense) as might be used in the technique of sports massage and so it is deemed to be quite safe for practitioners to use ${ }^{[17]}$.

\subsection{Conceptual framework}

The conceptual framework of this study relies mainly on the patient management framework and the key principles underpinning this framework are patient-centred care, safe and high quality care, multidisciplinary care, supportive care and care coordination. The framework developed by The Department of Human Services in Victoria, Australia ${ }^{[18]}$ with the emphasis on the improvement of patient outcomes by facilitating consistent care based on evidence and best practice, care pathways, clinical guidelines, standard and research that exist to ensure that patient and their families receive optimal care and support. The authors set out the key requirements for the provision of optimal care which need to be considered at each step of the care pathway and in this study the researchers will focus on the principle of safe and high quality care. Cancer care is complex, involving a range of clinicians with different experiences. To ensure safe and high quality cancer care, it is important that nurses should have the technical skills and experience to carry out FM postoperatively for cancer patients. Such care requires the support of a multidisciplinary team as a cornerstone of best practice in cancer pain management with efficient planning and collaboration all set within a context of encouraging further clinical trials and research in

holistic aspects of cancer care ${ }^{[18,19]}$. In order to develop an appropriate and effective care plan for the treatment and care of cancer patients, a regular meeting should be scheduled for all health practitioners in the MDT[Multidisciplinary Team] to understand the plan by each member and advise on the benefits of FM and its effect on pain relief. Such active involvement of the MDT greatly increases the desired outcome of active participation and facilitates best practice management of any aspect of evidence based care practice ${ }^{[20]}$. Additionally the use of a systematic approach to care provision must also include a strong commitment to audit of outcomes and therefore it is very important to emphasis the evaluation of the effects of FM on postoperative pain in breast cancer patients.

\subsection{Research hypothesis}

H1: Breast cancer patients receiving foot massage in postoperative period will have less pain than those who do not.

$\mathrm{H} 2$ : There is a difference in vital signs in the postoperative period among breast cancer patients receiving foot massage and those who do not. 


\section{Material and methods}

\subsection{Study design}

A quasiexperimental design was used to demonstrate causality between an intervention and an outcome. In this study the approach was used to estimate the impact of FM on postoperative pain and vital signs and its impact on pain intensity score. To be more certain, in this design the researchers may decide to have two groups: one experimental and one comparison. Also they measure the outcome on the experimental group before and after the intervention. In addition they carry out the same measurements with patients on the control group who did not receive the intervention. So there is a more confidence that a change has happened (if it has). Also this design lacks the element of random assignment but exercises certain controls and uses criteria other than random assignment (e.g. an eligibility cut-off mark) to enhance the study's internal validity and thereby strengthen the quality of evidence ${ }^{[21,22]}$. The study was conducted through the surgical department at the National Cancer Institute [NCI], Cairo University in Egypt from July 302013 to February 4, 2014. Consecutive sample was utilized and all eligible patients admitted to the surgical department who had undergone excision of a mass via simple mastectomy (BM) or a modified radical mastectomy (MRM) and who accepted to participate in the study were included. Sixty patients were included (30 in the experimental group and 30 in the control group). The inclusion criteria were that those involved were postoperative women with breast surgery, above 20 years of age and who pain intensity level $\geq 4$. Exclusion criteria were identified as those experiencing visual audible, or verbal communication difficulties; people with diagnosed mentally disability; hypertensive patients; those required blood pressure taken by foot; patients with thrombophlebitis, deep vein thrombosis or clotting disorders; those with varicose veins, inflammatory diseases or peripheral neuropathy.

\subsection{Technique for FM}

The purpose and procedure of FM was explained by the researcher to all participants who met the inclusion criteria. After obtaining written informed consent the technique of petrissage and friction is then applied to the feet. Petrissage is applied as direct pressure to the soft tissue underlying the skin in a slow and rhythmic fashion with the movement the balls of the fingers and thumbs with using classical massage techniques. Then friction is used through pressing the skin with smaller circular movements using the pad of the fingers. These movements facilitate an increase in blood flow and tissue and lymphatic circulation which removes lactic acid between the muscle fibers and reduces fatigue culminating in pain relief ${ }^{[23]}$. The message was given to the entire cohort of study participants in the experimental group by one co-investigator who had received a practical training course in the therapeutic use of FM before performing the procedure. Six hours after participants returned from the operating room their pain score was assessed by using a Visual Analogue Scale [VAS] and participants with score $\geq 4$ were included under the study and a session was introduced for 30 minutes in the form of 10 minutes providing reassurance and explaining the procedures and benefits of FM. This was then followed by a total of 20 minutes of foot massage, 10 minutes for each foot, in a comfortable bed and quiet environment. This technique was performed for the study participants in the experimental group while participants in the control group were in a separate room and received only standard care to relieve pain in the form of analgesics treatment. The pain intensity level was measured and recorded immediately before, 1 hour and repeated 2 hours after the massage for both groups. For both the experimental and control group analgesics were utilized based on study participants' request. Non-steroidal anti-inflammatory drugs (Perfelgan 1g IV every 6 to 8 hours and Xefo $8 \mathrm{mg}$ IM every 8hours) were used either solely or together with the opioid Pethidine (according to body weight, Pethidine was prescribed in the ratio of 50mg for patients with body weight less than $50 \mathrm{~kg}$ and from 75 to $100 \mathrm{mg}$ for body weight above $50 \mathrm{~kg}$ ). Patients in the control group received only analgesics, whereas those in the experimental group received FM with analgesic.

\subsection{Data collection}

The method of data collection was a structured questionnaire developed by the researcher first to elicit information related to the study participants' characteristics such as age, level of education, type of surgery and usage of NSAID. The second part of the questionnaire collected data related to assessment of pain intensity level utilizing the VAS as a reliable tool immediately before starting the FM and after 60 minutes and 120 minutes of foot massage ${ }^{[24]}$. Also vital signs were assessed using the same time intervals. Systolic and diastolic blood pressure were measured using deluxe mercurial 
sphygmomanometer where participants were lying on bed in the supine position. The pulse was measured by counting radial artery beats, and respiration was measured by counting thorax movements for a full minute using stop watch.

\subsection{Ethical considerations}

Institutional Review Board approval was obtained by the NCI in Egypt where the researchers were enrolled while completing the study. Individual participation in the study was voluntary. The consent form was reviewed with the participants prior to the start of the interview and signed accordingly. Confidentiality was maintained by keeping privacy of all participants' information.

\subsection{Data analysis}

The data were analyzed using statistical analysis with Statistical Package for Social Science for Windows (SPSS) Version 17. Statistical analysis was done using frequency, mean, SD and chi-square test, to compare participants characteristics in experimental and control group. The independent $t$-test was used for the comparison of each of the two time periods in both groups and the difference between pain intensity measurements of the control and experimental group. The significance level was established at $p<.05$. For variance analysis for repeated measurements, the Pillai trace test was utilized to compare pain intensity level and vital signs immediately before and at 1 hour after and 2 hours after the FM intervention.

\section{Findings}

The mean age of the study participants in the control group was $49.43 \pm 3.93$ years, whereas that of participants in the experimental group was $47.9 \pm 5.06$ years; however there was no statistically significant difference between both groups $\left(\chi^{2}=1.311 ; P=.195\right)$. Table 1 below demonstrates that there was no statistically meaningful difference between level of education and the use of analgesics among the control and experimental group $(P>.05)$. The same table illustrates that in each group $53.30 \%$ had undergone BM whereas $46.70 \%$ underwent MRM.

Table 1. Characteristics of participants in both groups

\begin{tabular}{|c|c|c|c|c|c|c|c|}
\hline \multirow[b]{2}{*}{ Characteristics } & & \multicolumn{2}{|c|}{ Control group } & \multicolumn{2}{|c|}{ Experimental group } & \multicolumn{2}{|c|}{ Chi-square test } \\
\hline & & No. & $\%$ & No. & $\%$ & $\chi^{2}$ & $P$-value \\
\hline Age & Mean \pm SD & \multicolumn{2}{|c|}{$49.43 \pm 3.93$} & \multicolumn{2}{|c|}{$47.9 \pm 5.06$} & 1.311 & .195 \\
\hline \multirow{4}{*}{ Level of education } & Can't read & 8 & $26.70 \%$ & 4 & $13.30 \%$ & \multirow{4}{*}{5.752} & \multirow{4}{*}{.124} \\
\hline & Primary & 6 & $20.00 \%$ & 8 & $26.70 \%$ & & \\
\hline & Second & 16 & $53.30 \%$ & 14 & $46.70 \%$ & & \\
\hline & Higher education & 0 & $0.00 \%$ & 4 & $13.30 \%$ & & \\
\hline \multirow[b]{2}{*}{ Use of Analgesics } & NSAID + Opioid & 17 & $56.70 \%$ & 14 & $46.70 \%$ & \multirow{2}{*}{0.601} & \multirow{2}{*}{.438} \\
\hline & NSAID & 13 & $43.30 \%$ & 16 & $53.30 \%$ & & \\
\hline \multirow[b]{2}{*}{ Type of surgery } & $\mathrm{BM}$ & 16 & $53.30 \%$ & 16 & $53.30 \%$ & \multirow{2}{*}{0.000} & \multirow{2}{*}{1.000} \\
\hline & MRM & 14 & $46.70 \%$ & 14 & $46.70 \%$ & & \\
\hline
\end{tabular}

Note. $\mathrm{BM}=$ Simple mastectomy; $\mathrm{MRM}=$ Modified radical mastectomy; NSAID = Nonsteroidal anti-inflammatory drugs

Table 2 indicated that there was a statistically significant difference of VAS after 1 hour among both groups $(P<.05)$. The same table showed reduction in pain intensity level from baseline to 1 hours 2.07 points $(6.90 \pm .061$, 1hour after receiving analgesic $4.83 \pm 0.83)$ in the control group and 3.10 points $(6.43 \pm 1.22$, 1hour after the massage $3.33 \pm 1.12)$ in the experimental group. The table also showed reduction in the pain intensity level from 1hour to 2 hours, 2.43 points $(4.83 \pm$ $0.83,2$ hours after receiving analgesic $2.40 \pm 0.77)$ in the control group and 1.43 points $(3.33 \pm 1.12$, 2 hours after the massage $1.90 \pm 0.71$ ) in the experimental group. Also a decrease of 4.53 points was indicated for the participants in the experimental group (baseline VAS is $6.43 \pm 1.22$, two hours after the massage $1.90 \pm 0.71$ ). 
Table 2. Comparison of the pain intensity levels using Visual Analog Scale [mean(SD)] of participants in control and experimental group

\begin{tabular}{lllllll}
\hline \multirow{2}{*}{ Follow-up Times, hr* } & Control group & \multicolumn{2}{l}{ Experimental group } & \multicolumn{2}{c}{ Independent $\boldsymbol{t}$-test } \\
\cline { 2 - 7 } & Mean & SD & Mean & SD & T & \multicolumn{1}{c}{-value } \\
\hline VAS baseline & 6.90 & 0.61 & 6.43 & 1.22 & 1.872 & .066 \\
VAS after 1hr & 4.83 & 0.83 & 3.33 & 1.12 & 5.869 & $* .000$ \\
VAS after 2hrs & 2.40 & 0.77 & 1.90 & 0.71 & 2.611 & .011 \\
\hline
\end{tabular}

Notes. When patients complained about pain, baseline means immediately before receiving analgesic and again 1 and 2 hours in control group, in sync with experimental group baseline means immediately before starting the foot massage when patients complained about pain.

VAS $=$ Visual Analog Scale; hr* $=$ hour; $* P \leqslant .001$

Table 3 showed that the mean pain intensity level in both groups decreases at all times and the greater reduction within the experimental group had a higher level of statistical significance $(F=46.550 ; P<.001)$.

Table 3. Comparison of pain intensity level over time, Mean \pm SD of patients in control and experimental groups

\begin{tabular}{llll}
\hline Follow up time hr* & Control (Mean \pm SD) & Experimental (Mean \pm SD) & Test \\
\hline VAS Baseline & $6.9 \pm 0.61$ & $6.43 \pm 1.22$ & \\
VAS After $1 \mathrm{hr}$ & $4.83 \pm 0.83$ & $3.33 \pm 1.12$ & $* 46.550$ \\
VAS After 2hrs & $2.4 \pm 0.77$ & $1.9 \pm 0.71$ & $* P<.001$ \\
& $F$-value $=23.87$ & F-value $=31.313$ & \\
& $P<.001$ & $P<.001$ &
\end{tabular}

Note. When patients complained about pain, baseline means immediately before receiving the analgesic and again 1 and 2 hours in control group, in sync with the experimental group baseline means immediately before starting the foot massage when patients complained about pain.

VAS $=$ Visual Analog Scale; hr* $=$ hour; ${ }^{*} P \leqslant .001 ; F=$ Variance analysis of repeated measurements, Pillai trace test.

Table 4 illustrated changes of vital signs over time among both groups and a statistically significant reduction of systolic and diastolic blood pressure in both groups but higher reduction was observed in the experimental group $(P \leq .001 ; F=$ 53.369- $F=32.112 ; P \leq .001$; respectively). The same table showed no statistically significant association was found between both groups as regards respiration $(F=5.279 ; P=.007)$.

Table 4. Comparison of vital signs over time, Mean \pm SD

\begin{tabular}{|c|c|c|c|}
\hline Follow up time, hour & Control group (Mean \pm SD) & Experimental group (Mean \pm SD) & Test \\
\hline Pulse Baseline & $73.2 \pm 1.19$ & $72.97 \pm 1.94$ & \multirow{5}{*}{$\begin{array}{l}F=10.600 \\
* P<.001\end{array}$} \\
\hline After $1 \mathrm{hr}$ & $72.4 \pm 1.33$ & $73.37 \pm 1.75$ & \\
\hline After $2 \mathrm{hrs}$ & $71.93 \pm 1.6$ & $72.17 \pm 0.87$ & \\
\hline F-value & 8.847 & 5.963 & \\
\hline P-value & 0.002 & 0.009 & \\
\hline Systolic blood pressure Baseline & $130.17 \pm 7.48$ & $128.1 \pm 6.43$ & \multirow{5}{*}{$\begin{array}{l}F=53.369 \\
* P<.001\end{array}$} \\
\hline After $1 \mathrm{hr}$ & $126.6 \pm 7.07$ & $126.5 \pm 5.96$ & \\
\hline After $2 \mathrm{hr}$ & $125.1 \pm 6.77$ & $123.97 \pm 5.38$ & \\
\hline F-value & 39.607 & 19.975 & \\
\hline P-value & $<0.001$ & $<0.001$ & \\
\hline \multicolumn{3}{|l|}{ Diastolic blood pressure } & \multirow{6}{*}{$\begin{array}{l}F=32.112 \\
* P<.001\end{array}$} \\
\hline Baseline & $79.97 \pm 3.93$ & $78.33 \pm 4.15$ & \\
\hline After $1 \mathrm{hr}$ & $79.17 \pm 3.78$ & $77.6 \pm 3.92$ & \\
\hline After $2 \mathrm{hrs}$ & $79.17 \pm 3.78$ & $76.77 \pm 3.9$ & \\
\hline F-value & 19.333 & 19.872 & \\
\hline$P$-value & $<0.001$ & $<0.001$ & \\
\hline Respiration Baseline & $17.77 \pm 0.97$ & $17.33 \pm 0.66$ & \multirow{5}{*}{$\begin{array}{l}F=5.279 \\
P=.007\end{array}$} \\
\hline After $1 \mathrm{hr}$ & $17.53 \pm 0.68$ & $17.27 \pm 0.64$ & \\
\hline After $2 \mathrm{hrs}$ & $17.27 \pm 0.64$ & $17.2 \pm 0.61$ & \\
\hline F-value & 5.579 & 0.563 & \\
\hline$P$-value & 0.005 & 0.567 & \\
\hline
\end{tabular}




\section{Discussion}

Around the world improvement in pain management among hospitalized patients is a high priority goal. Foot massage is an inexpensive and safe intervention when provided by an experienced therapist (nurses, physiotherapists or trainees) and on suitably assessed patients. The findings of this study showed that 20 minutes of FM intervention significantly reduced postoperative pain intensity levels within the first and second hour. Accordingly it is argued that the FM intervention can be safely and effectively incorporated into standard nursing practice in the postoperative care of patients recovering from breast surgery. This finding supports the effectiveness of FM as a modality to relieve postoperative pain. Findings in this study recorded that VAS readings 2 hours after the massage were also assessed to be lower than those one hour after the massage which is similar to a study conducted in Iran to evaluate the effectiveness of foot massage on postoperative pain in breast surgery patients which found that patients who were given a total of 20 minutes of FM perceived less pain after 120 minutes ${ }^{[11]}$. The same study also added that the intensity of pain in patients in the experimental group was very low at the 60 minutes mark compared with before the massage, a finding that is similar to our study findings. In this present study the researchers found a decrease of pain intensity level of 4.53 points in the experimental group after 2 hours after FM. Also pain intensity level of those in the control group decreased by an average of 4.5 points after 2 hours of receiving analgesics medication and this finding is supported by other studies carried out to evaluate the effectiveness of foot and hand massage in post cesarean pain control that reported a decrease in pain intensity level of 2.76 points for the participants under the study after applying foot and hand massage ${ }^{[23]}$. This finding is also consistent with a previous study conducted to investigate the effect of foot and hand massage as an intervention for postoperative pain which highlighted that pain intensity levels decreased from 4.00 to 1.88 after applying 20 minutes of foot and hand the massage ${ }^{[25]}$. Furthermore the same investigator highlighted that a reduction in pain perception after massage affects the sympathetic responses among post operative patients and thus supports the effectiveness of foot massage as an intervention to decrease postoperative pain ${ }^{[25]}$. In the same line ${ }^{[26,27]}$ other authors have published similar findings. In addition the findings of this current study are congruent with the findings of the study carried out to investigate the effect of foot and hand massage in post cesarean section and reported 90 minutes after the massage were assessed to be lower than those right after the massage ${ }^{[27]}$. Other researchers ${ }^{[23]}$ have published data suggesting that intensity of pain in the experimental group was very low at the 60 minutes mark compared with before the massage and after 90 minutes was found to be slightly higher than right after the massage but in our study pain intensity level after 2 hours was found to be lower than 1 hour after the massage. In addition other researchers have applied foot massage after abdominal surgery and found that the pain intensity level in the experimental group decreased more than that in the control group immediately after the massage ${ }^{[28]}$. On the other hand a study conducted in USA evaluated the effect of foot and hand massage on patients perception of care following laparoscopic sterilization as an intervention for postoperative pain reported no significant association of pain intensity scores from 5 minutes FM ${ }^{[29]}$, which suggested that the duration of massage may play an important role in its effect on postoperative pain. So the impact of massage duration and frequency on the efficacy of the massage in pain reduction after breast surgery needs to be more fully delineated in future studies. The gate control theory of pain reduction is considered one of several theories that have been proposed to explain the mechanisms underlying the effects of massage therapy as an analgesic because the pressure applied with massage travels down the nerve pathway faster than pain, blocking pain transmission $^{[13]}$.

Although we observed a significant reduction in patients reported pain in the experimental over the control group, we did not observe a significant reduction in the use of analgesic medication. However more patients in the control group requested NSAID plus Opioid than did those in the experimental group. This finding is incongruent with other studies conducted to determine the effect of foot and hand massage on post-cesarean section pain that reported a significant difference between study and control group in all types of analgesics including Diclophenac Sodium and Pethedine ${ }^{[27]}$.

It was further reported that performing FM resulted in a considerable decrease in the vital signs of the patients. In this current study, it is observed that a decrease in all vital signs among participants in the experimental group occurred after one and two hours following FM. In our study the differences between systolic and diastolic blood pressure readings among the experimental and control group were found to be significantly meaningful and these findings were similar with other study conducted to determine the efficacy of foot and hand massage on reducing postoperative pain where the 
researchers compared vital signs before, after 60 and 90 minutes of foot and hand massage and reported significant differences between experimental and control group ${ }^{[23]}$. In our study FM before and after 2 hours in the experimental group indicated a drop of $4.1 \mathrm{mmHg}$ in mean systolic blood pressure and $1.56 \mathrm{mmHg}$ drop in mean diastolic blood pressure; a drop of 0.8 beats per minutes in their mean pulse rate which were illustrated significant differences however a drop of 0.13 in their mean of respiratory rate per minute found no statistically significant difference. Other investigators published significant decreases in heart rate and respiratory rate after foot and hand massage as an intervention for postoperative pain, however no statistically significant differences were found in systolic and diastolic blood pressure after the massage ${ }^{[25]}$. On the other hand a crossover pilot study reported that a slight difference in the mean of participants' vital signs before and after foot massage with only a drop of $1 \mathrm{mmHg}$ in mean systolic blood pressure and $2 \mathrm{mmHg}$ drop in mean diastolic blood pressure; a drop of 3 beats per minutes in their mean pulse rate and a drop of 1 in their mean of respiratory rate per minute however no significant differences were found ${ }^{[30]}$. Similarly other investigators have reported that after each session of massage therapy on legs, arms, and face there was a direct decrease in systolic blood pressure ${ }^{[31]}$. In addition the same investigators found that changes in diastolic blood pressure were not seen until after 6 weeks of consecutive massage therapy but in our study we found a slight reduction in diastolic blood pressure in the experimental group after 1 and 2 hours of FM. The effects of massage on blood pressure may have physiological factors ${ }^{[32]}$.

In comparison to the initial measurements, all vital signs except respiration in the experimental group were found to be lower and within normal ranges in the measurements performed 2 hours later, which was considered to be statistically significant. Other investigators have reported a decrease in all vital signs of patients in the experimental group after 5 minutes of FM and failure of systolic and diastolic blood pressure and respiratory rates to again reach their pre-massage level after 120 minutes ${ }^{[11]}$. These researches have also added that pulse rate was decreased at 5 minutes mark and increased at the $30,60,90$, and 120 minutes marks but in our study the pulse rate in the experimental group was decreased in comparison with the initial measurement and after 1 and 2 hours of FM. The vital signs measurements in this study were used as objective parameters. As medical surgical nurses the researchers are aware of the present state of patients' pain and its concomitant effects on vital signs such as blood pressure and pulse, in the post operative period. So the findings of this study could potentially be useful if repeated in different settings to confirm the effect of FM on blood pressure.

\section{Limitation}

Massage was performed for all study participants by one trained massage nurse as a second investigator .Although this study showed a positive effect of FM in decreasing pain intensity level it cannot be generalized to all postoperative patients with breast cancer due to recruitment of a small sample size. The study was conducted in surgical wards that were very noisy with the frequent intrusion of nurses' interruption, visitors, and health care team members. A dedicated private room for conducting the massage was sometimes not available and thus the study findings might have been affected by these confounding variables potentially leading to a negative implication in a massage study.

\section{Conclusion}

The aim of this study was to evaluate the effects of FM on postoperative pain and vital signs in breast cancer patients. Based on the findings of this study, FM is suggested to be an effective technique in relieving pain level with a predictably higher rate of success in postoperative breast cancer patients. Implementing such technique was deemed to be feasible, nondisruptive, comforting and greatly appreciated by patients.

To enhance the nurses' role in quality improvement, nurses providing massage to the participants with postoperative pain should have sufficient experience with FM and in this study the co-investigator received training course before implementing the technique thus allowing for a consistent level of intervention. Also continuous developmental sessions on the technique of FM are provided for all nurses in the surgical departments at NCI in Egypt where brochures are prepared and distributed by the researchers to assist nurses to describing the technique of FM and the nurses' role in postoperative pain management. Using techniques such as FM is a very valuable adjunct to normal nursing care as it enhances the role of the nurse in pain relief using a safe, inexpensive, non-toxic technique that requires no special 
equipment, is easy to employ and has high efficacy. Additionally this noninvasive technique acts as a powerful non-pharmacological supportive method for patients whose pain is not adequately controlled by medication alone. So during the conducting of this study the continuing education department at NCI established a protocol detailing the nurses' role and participation in pain management using FM and encouraged up the surgical nurses to implement the FM technique based on scientific guidelines with a mature focus on proper assessment to negate its risks. Nursing staff also can spread awareness among the general population towards the usefulness of FM in relieving pain. Also FM can easily be taught to family members or properly selected unlicensed personnel so supporting the ongoing and continued benefits associated with the technique. Patients undergoing breast surgery receive tremendous benefits in health related quality of life. By recognising the challenges our patients face in the postoperative period such as pain and combining our need to bring evidence based technique such as FM to bear on such challenges, we can build on the solid foundation that surgery provides and improve the health and wellbeing of our patients.

\section{Recommendation}

This study has suggested that FM technique and its contraindications should be taught in the nursing curriculum under the umbrella of fundamentals of surgical nursing procedures so as to provide the practice with a more caring and holistic flavor. With the dramatic nature of the impact of breast surgery on the body especially among women, any intervention that safely mitigates the negative effects of pain is worthy of further consideration.

Further research studies are required to address the lack of standardization that arises from inconsistency of the duration of massage techniques. Study findings need to be replicated in different settings to confirm the efficacy of FM technique for pain management in postoperative patients and its impact on quality of life.

To support clinical relevancy of this study findings, further research needs to be carried out to investigate the relationship between FM and its impact on vital signs in postoperative patients. Observing benefits and the potential effects of FM is better enhanced by demonstrating more than one session to evaluate the clinical relevance of ongoing massage. So regular meeting should be held by health team members for discussing the outcomes, benefits and implementation of FM. Finally nurses have the abilities and skills to provide effective post operative pain management by applying FM to patients having no risks for receiving it.

\section{Acknowledgment}

Special thanks and gratitude are offered to the Head of the Department of Surgery and the Research Centre Team at NCI in Egypt for their cooperation and support during conducting this study. Special thanks and gratitude for Mr. Jerome Marley for his assistance in reviewing and editing the paper.

\section{References}

[1] Sommer M, DeRijke JM, VanKleef M, Kessels GH,and Peter ML. The prevalence of postoperative pain in a sample of 1490 surgical inpatients. European Journal of Anaesthesiology. 2008; 25(4): 25-34. PMid:18053314 http://dx.doi.org/10.1017/S0265021507003031

[2] Trish D, Christopher AM. Massage therapy: Integrating research and practice critical thinking, best evidence and future directions to advance the profession. (E book) Chester: Library of Congress. 2012 .Available at Human kinetics .com

[3] Gwen W. Team science of nursing, engineering, statistics, and practitioner in the development of a robotic reflexology device. Journal of the Society for Integrative Oncology. 2010; 8(1): 14-19.

[4] Zawilla N. Breast cancer in Egypt: A fact sheet. The Health Journal. 2011; (2)1: 8-10.

[5] Cassileth BR, Vickers AJ: Massage therapy for symptom control: Outcome study at a major cancer center. Journal of Pain Symptom Management. 2004; (28)1: 244-249. Available from: http://www.cancernetwork.com/integrative-oncology/massage-therapy\#sthash.mymjT8HV.dpuf. PMid:15336336 http://dx.doi.org/10.1016/j.jpainsymman.2003.12.016

[6] Ernst E. Massage therapy for cancer palliation and supportive care: a systematic review of randomized clinical trials. Journal of Supportive Care in Cancer. 2009; 17: 333-337 PMid: 19148685 
[7] Corbin L. Safety and efficacy of massage therapy for patients with cancer. Journal of Cancer Control. 2005; 12(3): 158-164.

[8] Sagar SM, Dryden T, Wong RK. Massage therapy for cancer patients: a reciprocal relationship between body and mind. Current Oncology. 2007; 14 (2): 45-56. PMid: 1891200 http:// www. ncbi.nlm.nih .gov/ pmc/articles/PMC1891200/

[9] Smeltzer SC, Bare BG. Textbook of medical surgical nursing. (12thed.).Philadelphia PA. Lippincott .2010.

[10] Chanif C, Wongchan P, Wimonrat C. Does foot massage relieve acute postoperative pain? Literature Review. Nurse Media Journal of Nursing. 2013; 3(1): 483-497.

[11] Ucuzal M, Kanan N. Foot massage: Effectiveness on postoperative pain in breast surgery patients. Online Journal of Pain Management Nursing. (2012) p1-8. Available from:www.sciencedirect.com /science/.S152 490421200038

[12] Copstead LE, Banasik JL. Textbook of pathophysiology (3rd ed.). Philadelphia.PA:Elsevier Saunders.2005

[13] Rose A, Barb W, Cynthia B. The Effects of Massage Therapy on Pain Management in the Acute Care Setting. Journal of International Journal of Therapeutic Massage and Bodywork. 2010 March; 3(1): 4-11.PMid: PMC3091428

[14] Richards J, Hubbert AO .Experiences of expert nurses in caring for patients with postoperative pain. Pain Management Nursing. 2007; (8): 17-24. PMid:17336866 http://dx.doi.org/10.1016/j.pmn.2006.12.003

[15] Faurot KR,Gaylord SA, Mann JD. Training family caregivers in hand and foot massage for hospitalized patients: Feasibility, challenges and lesson learned. Complementary Health Practice Review. 2007; 12(3): 203-226.

[16] Coelho HF, Boddy K, Ernst E. Massage therapy for the treatment of depression: A systematic review. International Journal of Clinical Practice. 2008; 62(2): 325-33. PMIid: 18081800

[17] Ingraham P. Deep friction massages therapy for tendonitis. A guide to a simple self-massage technique sometimes helpful in treating common tendonitis injuries like tennis elbow or Achilles tendonitis. 2012 November; http://saveyourself.ca/articles/frictions.php (2nd February 2014 last access)

[18] Metropolitan Health and Aged Care Services. Patient management framework. Breast tumor stream: A guide to consistent cancer care. Available from: www.health.vic.gov.au/cancer (29 January 2014 last access).

[19] Magee LR, Laroche CM, Gilligan D. 'Clinical trials in lung cancer: Evidence that a programmed investigation unit and a multidisciplinary clinic may improve recruitment. Clinical Oncology. 2001; 13(4): 310-311.

[20] National Breast Cancer Centre- 'Multidisciplinary meetings for cancer care: A guide for health providers', National Breast Cancer Centre, Camperdown, NSW, Australia. 2010 PP 3-10 Available from:

www.canceraustralia.gov.au/sites/default/files/publications/mdm-mdc-meeting-for-cancer-care_504af02d7368d.

[21] Gerrish K, Lacey A. The research process in nursing (6th ed).UK: Wiley Blackwell publishing.2010.

[22] Parahoo K.Using research principles, process and issues (2nd ed.), China: Palgrave Macmillan. 2006.

[23] Degirmen N, Ozerdogan N ,sayiner,D ,Kosgeroglu N, Ayranci U. Effectiveness of foot and hand massage in postcesarean pain control in a group of Turkish pregnant women.Applied Nursing Research. 2010; 23(3): 153-158. PMid:20643325 http://dx.doi.org/10.1016/j.apnr.2008.08.001

[24] Burckhardt CS, Jones KD. Adult measures of pain: The McGill Pain Questionnaire (MPQ), Rheumatoid Arthritis Pain Scale (RAPS), Short-Form McGill Pain Questionnaire (SF-MPQ), Verbal Descriptive Scale (VDS), Visual Analog Scale (VAS), and West Haven-Yale Multidisciplinary Pain Inventory (WHYMPI). Arthritis Rheum. 2003; 49: S96-104. http://dx.doi.org/10.1002/art.11440

[25] Wang H, Keck J. Foot and hand massage as an intervention for postoperative pain. The American Society for Pain Management Nursing. 2004; 4(2): 59-65. http://dx.doi.org/10.1016/j.pmn.2004.01.002

[26] Nixon N, Teschendorff J, Finney J, Karnilowicz W. Expanding the nursing repertory :The effect of massage in postoperative pain. The Australian Journal of Advanced Nursing. 1997; 14(3): 21-26. PMid:9180443

[27] Abbaspoor Z, Akbari M, Najar S. Effect of foot and hand massage in post-cesarean section pain control:A randomized control trail. American Society for Pain Management Nursing. 2013; PMid: 23352729.

[28] Kim J, Park K, The effect of foot massage on postoperative patients following abdominal surgery. Journal of Korean Academy of Adult Nursing. 2002; 14(1): 34-43.

[29] Hulme J, Waterman H, Hillier V.The effect of foot and hand massage on patients ' perception of care following laparoscopic sterilization as day case patients. Journal of Advanced Nursing. 1999; 30(2): 460-468. PMid:10457249 http://dx.doi.org/10.1046/j.1365-2648.1999.01101.x

[30] Ejindu A.The effect of foot and facial massage on sleep induction, blood pressure, pulse and respiratory rate: Crossover pilot study.Complementary Therapies in Clinical Practice. 2007; 13: 266-275. PMid:17950182 http://dx.doi.org/10.1016/j.ctcp.2007.03.008

[31] Aourell M, Skoog M, Carleson J.Effects of Swedish massage on blood pressure. Complementary Therapies in Clinical Practice. 2005; 11: 242-246. PMid:16290894 http://dx.doi.org/10.1016/j.ctcp.2005.02.008

[32] McNamara M, Burnham D, Smith C, Carroll D.The effects of back massage before diagnostic cardiac catheterisation.Alternative Therapy Health Medicince. 2003; 9: 50-7. PMid:12564351 